\title{
Lattice Boltzmann Modeling of Micro-Fluidic Devices
}

\author{
D. S. Clague
}

January 28, 2002

U.S. Department of Energy

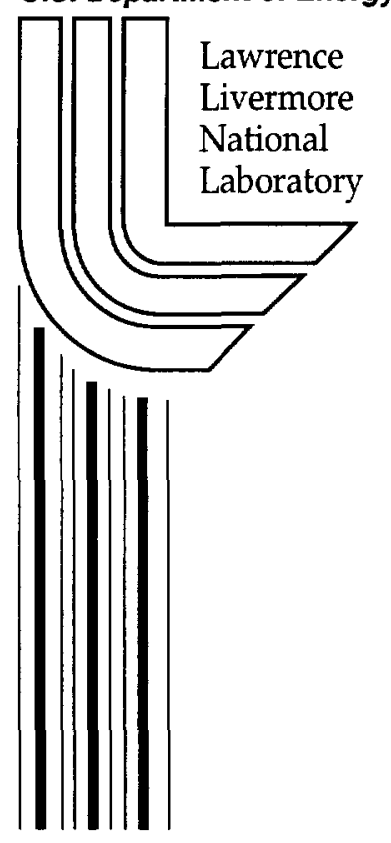




\section{DISCLAIMER}

This document was prepared as an account of work sponsored by an agency of the United States Government. Neither the United States Government nor the University of California nor any of their employees, makes any warranty, express or implied, or assumes any legal liability or responsibility for the accuracy, completeness, or usefulness of any information, apparatus, product, or process disclosed, or represents that its use would not infringe privately owned rights. Reference herein to any specific commercial product, process, or service by trade name, trademark, manufacturer, or otherwise, does not necessarily constitute or imply its endorsement, recommendation, or favoring by the United States Government or the University of California. The views and opinions of authors expressed herein do not necessarily state or reflect those of the United States Government or the University of California, and shall not be used for advertising or product endorsement purposes.

This work was performed under the auspices of the U. S. Department of Energy by the University of California, Lawrence Livermore National Laboratory under Contract No. W-7405-Eng-48.

This report has been reproduced directly from the best available copy.

Available electronically at http://www.doc.gov/bridge

Available for a processing fee to U.S. Department of Energy

And its contractors in paper from

U.S. Department of Energy

Office of Scientific and Technical Information P.O. Box 62

Oak Ridge, TN 37831-0062

Telephone: (865) 576-8401

Facsimile: (865) 576-5728

E-mail: reports@adonis.osti.gov

Available for the sale to the public from

U.S. Department of Commerce

National Technical Information Service

5285 Port Royal Road

Springfield, VA 22161

Telephone: (800) 553-6847

Facsimile: (703) 605-6900

E-mail: orders@ntis.fedworld.gov

Online ordering: http://www.ntis.gov/ordering.htm

OR

Lawrence Livermore National Laboratory

Technical Information Department's Digital Library

http://www.llnl.gov/tid/Library.html 


\title{
Lattice Boltzmann Modeling of Micro-fluidic Devices
}

\author{
by
}

David S. Clague, EE/EETD

\subsection{Motivation}

Many fluid based processes, e.g., in the chemical and pharmaceutical industry, are making the transition from macroscopic, bench scale laboratory instruments to micro-chip based devices much like the transition that occurred in the electronics industry more than a decade ago. This revolutionary transition represents the future in many important technologies including chemical and biological warfare detection systems, medical diagnostic instruments, the Human Genome project, and drug discovery applications. All of these technologies involve the transport of complex fluids in micron to millimetersized channels etched in microchip-like substrates. Instruments or devices that make use of such fluid processes are now known as micro-fluidic devices. An essential aspect of these systems is the manipulation of macromolecules such as DNA, or particles such as cells or spores, within a suspending fluid. At the micron length scales, forces that act on short length scales become important. As a result, we are developing simulation capabilities to study coupled physical effects.

A primary feature of these systems is the manipulation of macroscopic solutes within a suspending fluid. In these systems, colloidal particles and macromolecules experience a number of physical forces, which influence their transport and separation within microchannels. In the design of these systems, it is critical to understand and be able to creatively make use of these interactions and the resulting phenomena to achieve the desired device functionality. In a practical sense, the device manufacturing process from concept to prototype is becoming increasingly more time consuming and expensive; hence, the modeling and simulation aspect of micro-fluidic device design is now recognized by many as the critical need to guide current and future efforts in this area.

Traditional modeling approaches have included Finite Element Analysis (FEA) and Boundary Element Methods (BEM). FEA has been used to study the pure fluid behavior in microfluidic devices. A major limitation, however, is that it is very difficult to introduce "mobile" particles under the influence of external fields into an FEA simulation. Very few researchers have this capability built into their FEA codes. BEM have also been used to simulate pure fluid flow in these devices. BEM can more easily incorporate mobile particles than FEA but this approach is computationally expensive. Additionally, it is based on Stokes equations and therefore cannot handle finite Reynolds number flows or non-Newtonian fluid effects.

The Lattice Boltzmann method does not have these limitations. This approach can easily handle bounding walls, mobile particles, external fields, and finite Reynolds numbers. In this research effort, we have developed enhanced lattice Boltzmann (LB) simulation capabilities to study these complex fluid systems. Essentially, the LB method, solves a discrete form of the Boltzmann transport equation on a lattice. Additionally, the Lattice Boltzmann (LB) method is based on local interactions. As a consequence, it is easily parallelized relative to the conventional methods mentioned above. I have extended the capabilities of an existing LB code (developed by the PI) to model particle-laden fluid behavior in microfluidic devices, and verified the enhanced code with experimental 
results. The challenging aspects of this work are the incorporation of realistic representations of macromolecules, non-Newtonian fluid effects, the parallelization of the three-dimensional code, and the inclusion pair-wise interactions, i.e., interactions between macromolecules, and interactions between macromolecules and micro-channel walls.

\subsection{Scope}

The modeling and simulation aspect of micro-fluidic device design is now recognized by the National Laboratories, academia, and private industry, as a critical need to support ongoing and future efforts in this area. The experimental efforts in this rapidly growing and changing field have been guided primarily by good engineering intuition and by numerical algorithms not fully equipped to account for all of the complexities of such devices. Because these devices involve near field hydrodynamic and non-intuitive, non-hydrodynamic interactions, rigorous modeling and simulation tools need to be developed. The experimental frontiers in this area are constantly challenged and pushed. Micro-device functionality has expanded from simple electro-osmotic pumping on glass and silicon microchips to the dielectrophoretic DNA trap, which was develop through the LDRD program at LLNL by Robin Miles. As a result, the device manufacturing process from concept to prototype is becoming increasingly more time consuming and expensive. As discussed above, the existing modeling efforts help but provide an incomplete description of the observed phenomena. The LDRD ER project described here addresses the above modeling and simulation needs, and will result in a design tool, which can be applied to numerous microfluidic systems currently being worked on in several programs. Furthermore, the modeling and simulation capability will also give us insight into the physics of particle behavior in micro-flows, which has lead to the conception of new devices. This effort aligned with the Center for Microtechnology, the Center for Computational Sciences, LLNL's mission countering weapons of mass destruction (CW/BW detection work), BBRP's Human Genome Project work, the Medical Technology Program and the Joint Program Office (medical diagnostic instruments).

\subsection{Approach}

In the LB method, one solves the Boltzmann transport equation in a discrete fashion. In the limits of near equilibrium behavior, small Knudsen number, and small Mach number, one can recover the Navier-Stokes equations from the LB equation. Essentially, the "lattice" is a symmetric equi-spaced finite difference mesh. At each lattice site, the fluid is accounted for in a probabilistic manner through the single, fluidparticle velocity distribution function. By imposing the appropriate boundary conditions, the fluid is evolved on the lattice, and the desired fluid information is extracted. To account for finite-sized solutes, such as, macromolecules, one only needs to track the center of mass of the solute and to assign the appropriate lattice sites as solid phase. This approach enables one to track the particles dynamic position and to calculate the hydrodynamic forces acting on it; however, to properly account for the force interactions, the solute needs to have at least a few lattice sites between itself and the object it is interacting with. Additionally, because the LB method uses a discrete representation of the solute surface, the surface geometry is accounted for in a first order sense, i.e., the surface appears jagged unless a large number of lattice sites are used. The PI has incorporated a new approach that appropriately corrects the force for near-field solute 
interactions within one lattice site. This approach also corrects the discrepancy between the desired and the realized, discrete solute surface geometry. This same approach is required to incorporate the near-field, non-hydrodynamic interactions relevant to microfluidic devices. Previous attempts by other researchers to incorporate near-field hydrodynamic interactions were approximations. The work proposed here rigorously accounts for near-field hydrodynamic interactions. Simulation test cases for fluids through complex media, spherical solute-solute interactions, and solute-wall interactions agree well with existing theory.

\subsection{Technical Accomplishments}

Many microfluidic design tools only take into account the fluid behavior. The specific focus of this work was to take into account the details of the microstructure and in particular, the suspended, mobile phase, which includes target species. Therefore, our aim in developing this capability was to enable device designs to predict how various design modifications would affect the transport behavior of the target species.

The strength of the lattice Boltzmann method is its flexibility in the description of complex microstructures. This strength applies to both stationary and mobile media. The weakness of this approach is that it is an explicit method, that is, hydrodynamic interactions evolve in time. Therefore, to make this capability a useful tool, advancements need to be made to speed-up simulations to provide analysis in a timely fashion. To achieve this increased efficiency in computational speed the capability was parallelized. In addition to being made parallel, we developed the capability to incorporate external field effects, non-Newtonian fluid models and bead-n-spring representation of macromolecules. During the LDRD effort, we used these new capabilities to assist device designers in determining the hydrodynamic force and torque on species held stationary by a dielectrophoretic force; the hydrodynamic lift effects observed in microflow conditions; and dynamics of dielectrophoretic capture.

\subsection{Features of the Capability}

\subsubsection{Code Parallelization}

The 3D lattice Boltzmann code has been modified to run on parallel computers. Because the lattice Boltzmann (LB) method has it's foundations in Cellular Automata (CA), like CA, the LB method is naturally designed for parallel computing. More specifically, all of the fluid properties are calculated locally at each lattice site; therefore, one can easily, in principle, parallelize down to the individual lattice site in this approach. In contrast, when studying the transport properties of mobile macro-particles, it is necessary not only parallelize the fluid but the mobile species as well. Because the fluid phase is accounted for at each lattice site, it was relatively straightforward to parallelize. This aspect was not trivial but was accomplished. For the work performed here we parallelized both the fluid and the mobile species. To handle the mobile, we therefore developed parallel algorithms to track mobile species as they traverse parallel, computational zones and to account for the hydrodynamic disturbances they produce in each zone. As a result, both the stationary macro-particle code and mobile macro-particle codes are parallel.

Once made parallel, the 3D stationary particle LB code was used to explore the hydrodynamic force and torque acting on a macro-particle trapped or held fixed near the surface of a microchannel. This question was posed by device designers who were using non-uniform electric fields to preferentially pull target species out of solution and trapping them near the surface of the channel, see Fig. 1 below. 


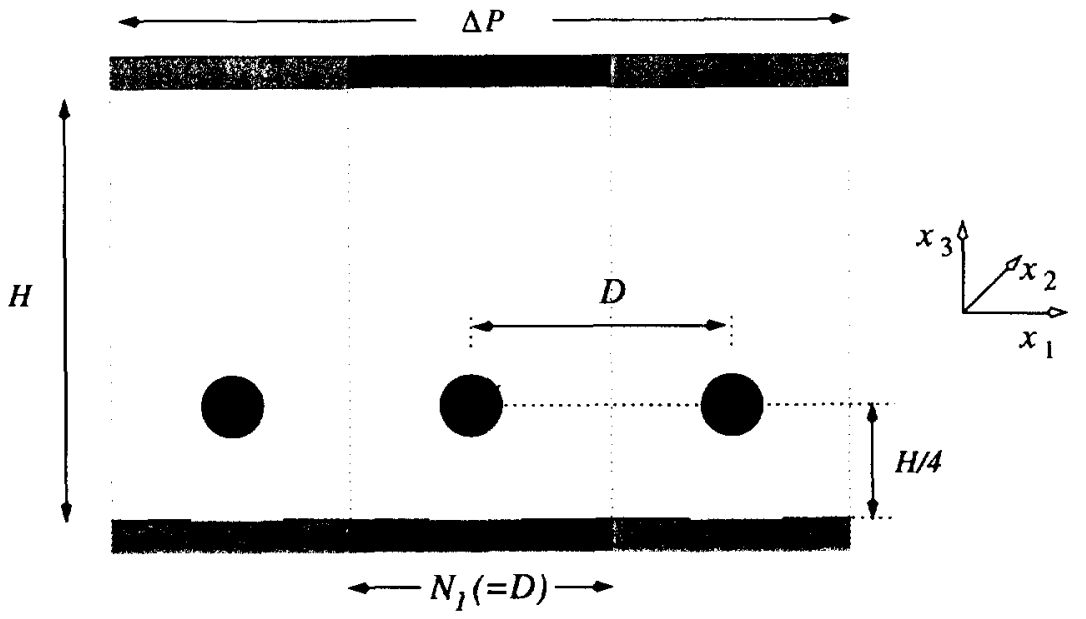

Figure 1. A test sphere of radius a at a height of $\mathrm{H} / 4$ is in the central flow cell which has vertical gap between the bounding walls of $H$ with a length of $N_{1}(=D)$ in the $\mathrm{x}_{1}$ and $\mathrm{x}_{2}$-directions. The periodic images in the $\mathrm{x}_{1}-\mathrm{x}_{2}$ plane are shown to be a distance $D$ away from the central sphere. The goal rectangles on the channel surface represent electrode locations.

Shown above in Figure 1 is a single sphere in flanked by it's periodic images in the $x_{1}-x_{2}$ plane. The images that extend into and out of the page, which are not shown, are also a distance $D$ from the central sphere. We are interested in calculating the hydrodynamic force and torque acting on the central sphere. The LB results are compared with the theoretical results for the same configuration (Happel and Brenner, 1991). With this capability, we were able to study the effects the distance between neighboring trapped particles and height of the channel, $H$, with fixed distance between neighboring trapped particles. Shown below in Figure 2 is the force the fluid exerts on the sphere as a function of distance between captured particles, $D$.

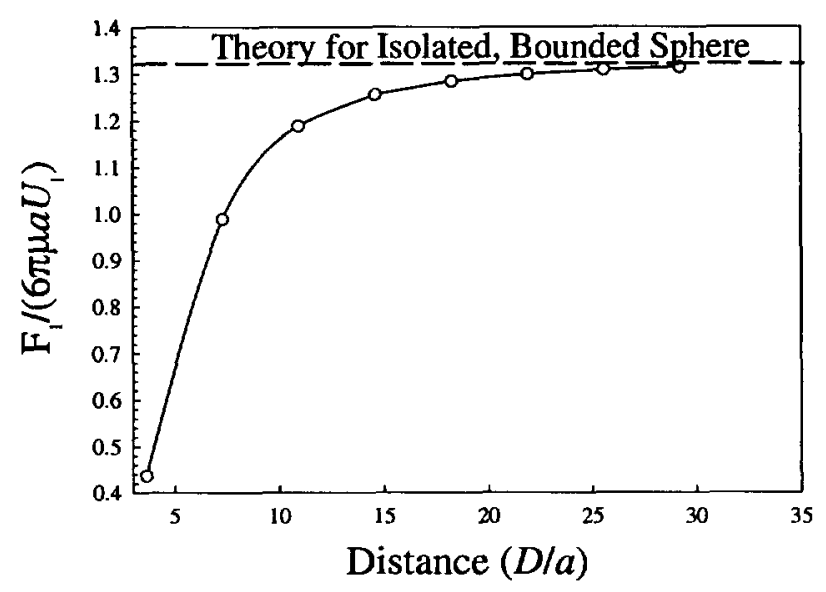


Figure 2. Force Acting on a Bounded Periodic Sphere. LB results are compared with theory. The dimensionless hydrodynamic force in the $\mathrm{x}_{1}$ direction, $F_{1}$, acting on the central sphere in Figure 1 is plot as a function of the dimensionless, center-to-center distance, $D / a$, between periodic images. The vertical position of the sphere is fixed at $\mathrm{H} / 4 . \mathrm{U}_{\ell}$ is the fluid velocity evaluated at $H / 4$.

As shown above, the force calcuated from the LB method is made dimensionless with Stokes drag force where $U_{\ell}$ is the fluid velocity evaluated at $H / 4$. Additionally, the center to center distance between the test sphere and it's periodic images, $D$, is made dimensionless with the sphere radius, $a$. The hydrodynamic force increases with increasing separation between periodic images. This is consistent with what Dabros and van de Ven (1992) observed for two aligned stationary spheres in a simple flow field directed along the line of centers. As they note, the test sphere experiences a screened hydrodynamic force. In their work, they also predict the test sphere will fill the full hydrodynamic force at sphere-sphere separation of approximately 30 sphere radii. This predicted reduced drag force is very relevant to device designers. For all of the results of this study the interested reader is directed to Clague and Cornelius (2000).

The parallel, 3D LB capability was also used to study mobile spheres in micoflow conditions. In particular, the mobile particle code was used to a study hydrodynamic lift force that was causing macro-particles to center themselves as they convected down stream with the fluid. To explore this lift phenomenon and to potentially learn how to exploit it, the LB capability was used to characterize particle behavior in rectangular microchannel flows. Shown in Figure 3 below are results for a study of lift as a function of channel Reynolds number.

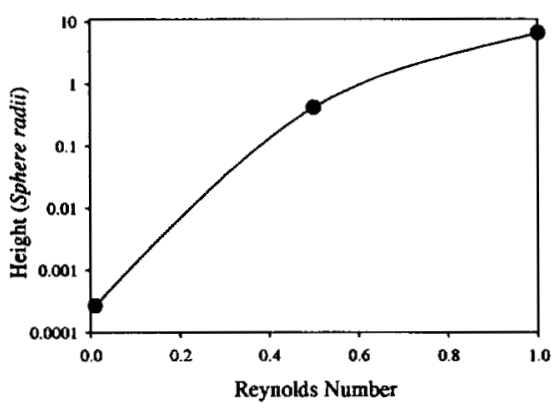

Figure 3. Hydrodynamic lift as a function of Reynolds Number. The lift height has been made dimensionless by the sphere radius, and the range of Reynolds numbers is less than or equal to 1 .

In Figure 1a, the lift height is made dimensionless with the sphere radius, and the Reynolds number is based on the maximum fluid velocity. According to Saffman's theory (1965), the lift force is proportional to the magnitude of gradient in the fluid velocity profile. As the Reynolds number increases, the gradient in the fluid velocity steepens, and the theory predicts an increase in the lift force. Typical Reynolds numbers range from much less than 1 to approximately 1 in microfluidic systems. As expected, the LB simulations capture the weak inertial forces and predict an increase in lift with an 
increase in the Reynolds number.

\subsubsection{Inclusion of External Fields}

In all of the physical systems of interest, external fields are present. Gravity is particularly important because the suspended species in the flow field typically experience buoyancy effects. Also, as a said goal of this LDRD effort, we proposed to be able to model and simulate the dielectrophoretic separations. Both gravity and the dielectrophoretic fields are electromagnetic in nature and therefore propagate orders of magnitude faster than hydrodynamics interactions. In addition, we're applying the external field effects to the suspended particles as apposed to the suspending fluid. Consequently, these fields effects were incorporated from continuum solutions and applied directly to the suspended particles. To accomplish this, the continuum forces were scaled from real space forces to lattice space forces and then applied directly to the suspended species. If however we were interested in applying these forces to the fluid phase, we would have to modify the collision operator in the LB formulation, but because we apply these forces directly to the suspended species, we do not modify the collision operator.

\subsubsection{Dielectrophoresis}

Dielectrophorsis (DEP) is a phenomenon by which a non-uniform electric field induces a net force on a polarizable particle (or macromolecule). As described by Pohl the field can be produced by either a direct current (DC) or alternating current (AC). The only requirement is that the field be non-uniform. This field non-uniformity causes an imbalance in the Lorenz forces produced at the (natural or induced) particle poles so that the resultant net forces is non-zero and causes particle motion. Depending on the relative difference between the complex dielectric properties of the suspended particle and the suspending medium, this net force causes particles to be either attracted or repulsed from regions of high field intensity. The use of dielectrophoresis is fast becoming a proven technique for manipulating particles and macromolecules in microfluidic systems. Through this LDRD effort, we were able, for the first time, to develop a dynamic simulation capability to study the capture of mobile particles in a dielectrophoretic separator. To achieve this result, it was required to develop a theoretical solution for nonuniform AC-field and the associated gradients in the electric field intensity. The analytic solution was employed to study the effects of electrode spacing and electrode width on the gradient in electric field intensity. Shown in Figure 4 are the components in the electric field intensity produced by an array of equi-spaced interdigitated electrodes.
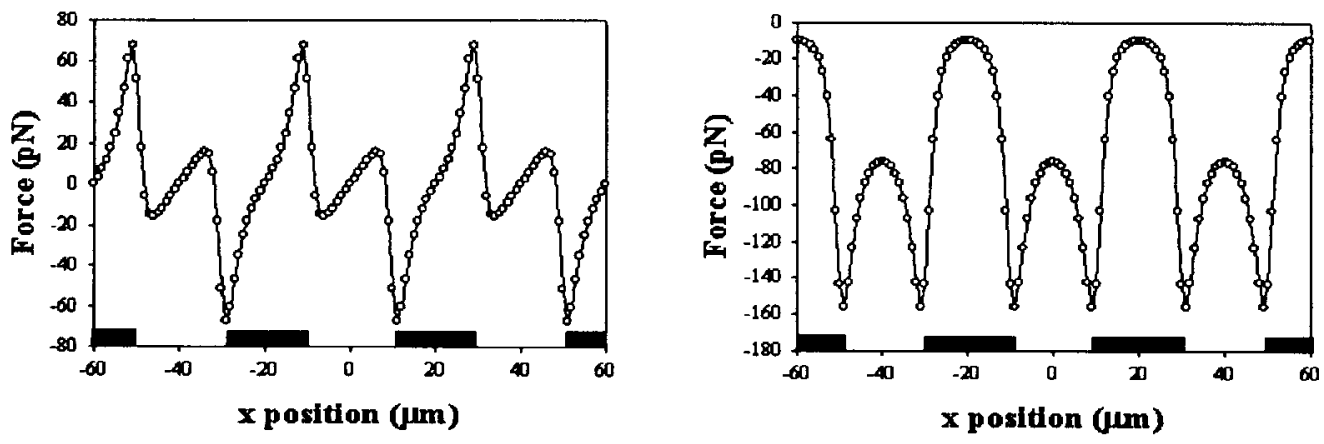
Figure 4. The $\mathrm{x}$ and $\mathrm{z}$ - components of the gradient in the electric field intensity produced by a parallel array of electrodes. The applied voltage is 5 Vrms and the electrode (electrodes shown in gold) width and spacing is set at $20 \mu \mathrm{m}$.

Results are summarized in the refereed paper in Physical Review E. (Clague and Wheeler, 2001). The dielectrophoretic force acting on the suspended species was incorporated as described in section 4.1.2, and through this LDRD effort, for the first time, the dielectrophoretic capture of a freely translating sphere with rigorous hydrodynamic interactions was demonstrated, see Figure 5.

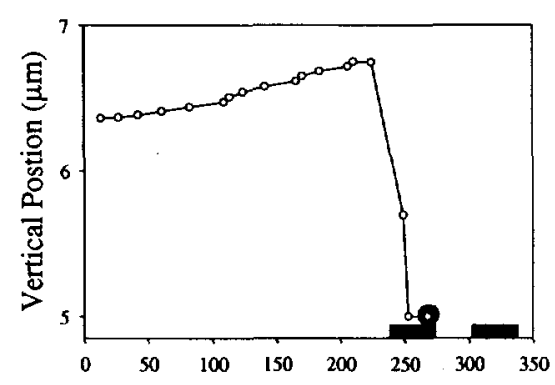

Figure 5. Trajectory of a spherical particle undergoing Dielectrophoretic capture. The sphere radius is $5 \mathrm{~mm}$; Reynolds number is $\sim 4$; and the applied voltage is $5 \mathrm{~V}_{\text {rms }}$.

In Figure 5, we have built in a time-averaged electric field into the LB capability to study coupled hydrodynamic and DEP forces on suspended species. As shown, the sphere initially experiences inertial lift forces, however, when it encounters the electrode array, it is pulled to the electrode surface and captured.

This result laid the foundation for additional studies in characterizing the threshold for DEP particle capture/trapping as a function of flow rate, applied voltage, particle radius and initial particle height. Furthermore, this capability enabled the PI to successfully compete for DARPA funds.

\section{Non-Newtonian Fluids}

The LB capability is now applicable to a broader class of bio-fluidic systems that is directly applicable to sample preparation and detection for biomedical and biotechnology applications. In Microfluidic devices, fluids, beads and other biological matter are transported through networks of micron-sized channels for sample preparation, separations and assays. When dealing with pathogens and toxins, understanding the transport behavior of target species in these devices is of paramount importance. The transport behavior is not only influenced by the colloidal interactions, but at these small length scales the shear rate, which gives rise to non-Newtonian fluid effects in bio-fluids, 
can be quite high; furthermore, the particle mobilities are clearly effected by their geometric features. Therefore, to better understand the influence of non-Newtonian fluid effects and particle geometry, the capability has been enhanced to handle power-law fluids (many bio-fluids exhibit shear thinning behavior) and bead-and-spring representation of species.

As described above, biofluids tend to have shear thinning behavior, that is, the fluid viscosity depends on the local shear rate. As a result, particle mobilities are significantly affected and exhibit non-intuitive behavior. To incorporate this behavior, the collision operator in the lattice Boltzmann formulation was modified to take into account local changes in the shear rate. Results from the new, enhanced lattice Boltzmann capability are compared with theory below in Figure 6.

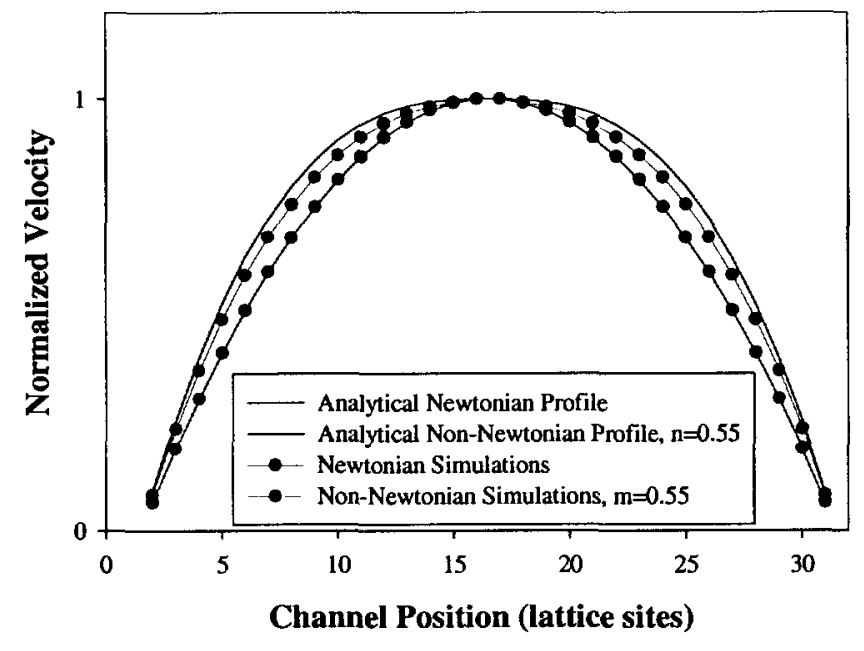

Figure 6. Lattice Boltzmann prediction of power-law fluid behavior compared with theory.

In Figure 6, the shear thinning and thickening behavior as predicted by the enhanced lattice Boltzmann capability are compared with power-law fluid theory for flow between parallel plates. As shown above, the simulation results are in good agreement with theory.

\section{Complex particle features}


As discussed above the lattice Boltzmann simulation capability has been enhanced to account for more complex particle features. Many species of interest, e.g., DNA strands, are best represented as long chains; hence, one aim of this year's effort was to develop a bead-n-spring representation of macromolecules, see Figure 7.

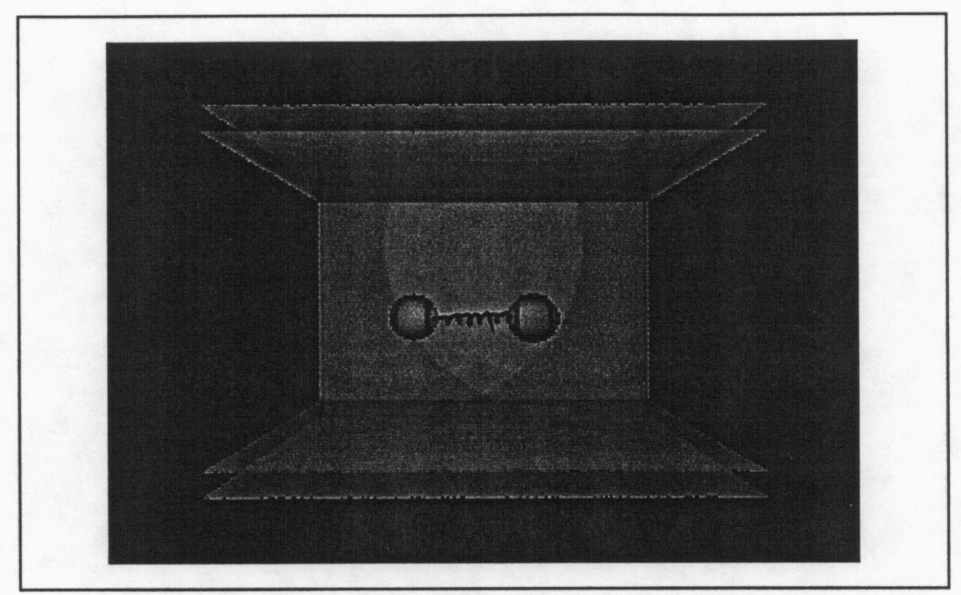

Figure 7. (Finite-extension-non-linear-elastic) The FENE-Dumbbell a building block for Bead-n-Spring representation of macromolecules.

As shown in Figure 2, we use the FENE-Dumbbell (Hur and Shaqfeh, 1997) model as a building block for representing long-chain macromolecules in the LB simulation capability. This model not only gives the desired representation of a macromolecule but the "spring" bridging the beads also causes the bulk fluid to have non-Newtonian character (Binous and Phillips, 1998).

\section{Successful Exit Plan}

DARPA recently launched a $\$ 50 \mathrm{M}$ program aimed at developing simulation tools for Mixed Technology Microdevices (micromachined, optoelectronic, and microfluidic). The PI was able to successfully demonstrate the utility of the Lattice Boltzmann technique in assisting the design of microfluidic devices, and secured DARPA funding in the amount of $\$ 900 \mathrm{~K}$ over 2 years.

\section{Conclusions}

The results to date do indeed show that the lattice Boltzmann method accurately solves relevant, non-trivial flow problems. The parallelization of both the fluid and the mobile species in flow has enhanced this capability such that it is useful for solving relevant problems in a timely fashion. The initial studies of stationary or capture species revealed evidence of hydrodynamic screening between upstream and downstream particles. Numerical studies reveal that the critical length for which the test particle is hydrodynamically decoupled from upstream and downstream particles is on the order of 30 sphere radii. For mobile species, the LB capability was shown to be naturally suited for predicting the hydrodynamic lift phenomenon (inertial lift). A conversion factor was 
developed based on scaling arguments to include relevant forces generated by external fields. Using this conversion, an analytic solution for the Dielectrophoretic force was included into the LB capability which enabled the study of Dielectrophoretic particle capture. The Non-Newtonian enhancements have expanded the applicability of the LB capability to more physical systems. Specifically, with the bead-n-spring representation of macromolecules researchers will be able to study chain dynamics in micro-, physiological and Bio-MEMS environments. Furthermore, the ability to capture the shear thinning behavior, without any increase in computational time, positions this capability to be applied to a whole host of new problems involving biofluids.

\section{References}

Dabros T, and van de Ven TGM "Hydrodynamic interactions between two spheres near a solid plane." J of Multiphase Flow 199218 (5) p 751.

Saffman P.G. "The lift on a small sphere in a slow shear flow," J. Fluid Mech (1965), vol 22 , part2, pp. 385-400.

Hur, J.S. and E.S. Shaqfeh "Brownian dynamics simulations of single DNA molecules in shear flow," J. Rheol. 44(4) 2000

Binous, H. and R. J. Phillips "Dynamic simulation of one and two particles sedimenting in viscoelastic suspensions of FENE dumbbells," J. Non-Newtonian Fluid Mech. 83, 93-130, 1999.

Happel and Brenner, "Low Reynolds Number Hydrodynamics," Kluwer Academic Publishers, 1991.

\section{Publications and Presentations Resulting From this Effort}

David Clague, "Lattice Boltzmann Simulation of Complex Fluids," Center for Computational Engineering thrust area report.

David Clague, "Lattice Boltzmann Simulation of Complex Fluids," Engineering Seminar Series, Nov. 1998 (sponsored by Robert Langland).

David Clague, "The hydrodynamic force and torque acting on a bounded, stationary object, presented at Small talk ' 99 ' in June.

David Clague, "The hydrodynamic force and torque acting on a bounded, stationary object, presented at Tri-Lab '99.'

David Clague, “Transport Properties of Macromolecules in Microfluidic and Physiological Systems, Invited talk, '00.' 
Dynamic Simulation of Macromolecules and Colloidal Particles in mflows, Presented at AIChE Annual meeting Clague, Wheeler, Hon, Radford, 2000

Clague "Lattice Boltzmann Simulation of Particle-Laden Flows in Microfluidic Devices," presented at the CMT ' 01 ' review.

Weisgraber, T H, Wheeler, E K and Clague D S, "Dynamic Simulation of Fluids and Suspensions in Microflows," presented at the 2001 AIChE national meeting.

Clague, D.S.; Wheeler, E.K. "Dielectrophoretic Manipulation of Macromolecules: Part 1 The Electric Field”. Phys. Rev. E, vol.64, (no.2), Aug. 2001

David Clague and Peter Cornelius, "A numerical study of the hydrodynamic force and torque acting on a bounded spherical body in Poiseuille flow," to appear '00,' International Journal for Numerical Methods in Fluids.

Garcia, M. and D.S. Clague, The 2D electric field above a planar sequence of independent strip electrodes, to appear '00,' J. of Applied Physics. 